\title{
Article \\ A Luminous Efficiency-Enhanced Laser Lighting Device with a Micro-Angle Tunable Filter to Recycle Unconverted Blue Laser Rays
}

\author{
Xinrui Ding ${ }^{1}$, Ruixiang Qian ${ }^{1}$, Liang $\mathrm{Xu}^{2}$, Zongtao $\mathrm{Li}^{1,3}{ }^{1}$, Jiasheng $\mathrm{Li}^{1}$, Caiman $\mathrm{Yan}^{1}$ and Binhai $\mathrm{Yu}^{1, *}$ \\ 1 National \& Local Joint Engineering Research Center of Semiconductor Display and Optical Communication \\ Devices, South China University of Technology, Guangzhou 510640, China; dingxr@scut.edu.cn (X.D.); \\ rxiang_q@163.com (R.Q.); meztli@scut.edu.cn (Z.L.); jiasli@foxmail.com (J.L.); chamenyan@163.com (C.Y.) \\ 2 Institute of Semiconductor Science and Technology, South China Normal University, 55 Zhongshan Avenue, \\ Tianhe District, Guangzhou 510631, China; xuliang@nationstar.com \\ 3 Guangdong Provincial Key Laboratory of Semiconductor Micro Display, Foshan Nationstar Optoelectronics \\ Company Ltd., Foshan 528000, China \\ * Correspondence: mebhaiyu@scut.edu.cn
}

check for

updates

Citation: Ding, X.; Qian, R.; Xu, L.;

Li, Z.; Li, J.; Yan, C.; Yu, B. A

Luminous Efficiency-Enhanced Laser

Lighting Device with a Micro-angle

Tunable Filter to Recycle Unconverted Blue Laser Rays. Micromachines 2021, 12, 1144. https://doi.org/10.3390/ mi12101144

Academic Editor: Hieu Pham Trung Nguyen

Received: 25 August 2021

Accepted: 21 September 2021

Published: 23 September 2021

Publisher's Note: MDPI stays neutral with regard to jurisdictional claims in published maps and institutional affiliations.

Copyright: (c) 2021 by the authors. Licensee MDPI, Basel, Switzerland. This article is an open access article distributed under the terms and conditions of the Creative Commons Attribution (CC BY) license (https:// creativecommons.org/licenses/by/ $4.0 /)$.

\begin{abstract}
In this work, a phosphor converter with small thickness and low concentration, based on a micro-angle tunable tilted filter (ATFPC), was proposed for hybrid-type laser lighting devices to solve the problem of silicone phosphor converters' carbonizing under high-energy density. Taking advantage of the filter and the scattering characteristics of microphosphors, two luminous areas are generated on the converter. Compared with conventional phosphor converters (CPCs), the lighting effects of ATFPCs are adjustable using tilt angles. When the tilt angle of the micro filter is $20^{\circ}$, the luminous flux of the ATPFCs is increased by $11.5 \%$ at the same concentration; the maximum temperature (MT) of ATFPCs is reduced by $22.8 \%$ under the same luminous flux and the same correlated color temperature (CCT) $6500 \mathrm{~K}$. This new type of lighting device provides an alternative way to improve the luminous flux and heat dissipation of laser lighting.
\end{abstract}

Keywords: hybrid type laser lighting; micro-angle tunable filter; heat dissipation

\section{Introduction}

Because of its excellent energy density, slope efficiency and brightness, laser light is in wide use in headlights, high resolution display [1-4]. For example, in high-resolution, intelligent headlights, spatial light modulators such as digital micro-mirror device (DMD) or liquid crystal display (LCD) provide enough high luminous flux to ensure the brightness of certain pixels. Such laser lighting systems have great potential [5,6], and researchers are committed to the continuous development of laser sources and phosphor converters.

To package and protect the phosphor, it is proportionately dispersed in the matrix [7], which has phosphor powder bonding material that includes polymers (e.g., epoxy resin and silicone) and inorganic materials (e.g., glass, ceramics, and single crystals) [8,9]. Based on these materials, considerable research has been carried out on improving luminous flux, uniformity of the correlated color temperature and temperature stability [10-15].

To improve the luminous flux or extraction efficiency of a solid-state lighting system, some researchers in the fields of laser and light emitting diode (LED) lighting are committed to reducing total internal reflection loss and Fresnel reflection loss by structured surfaces or microstructures. Ding et al. [16,17] processed roughened V-grooves with controllable shapes, angles and positions on chip-on-board substrates and packaging layers. Compared with traditional chip-on-board structures, those with V-grooved structures had an increased photoluminescence efficiency (PE) of 31.9\%. Pan et al. [18] analyzed the influence of pyramid microstructures on the optical efficiency of luminous elements and found that, after optimizing the density and angle of the pyramids, the PE of the chip increased from 
9.04 to $17.72 \%$. Similar strategies were also used on converters $[19,20]$. In addition to the structured surface and microstructures, some optical films or coatings were used to enhance the use of exciting rays to improve the PE in the previous works. Tang et al. [21] applied dichroic filters to the upper and lower surfaces of polymer-based phosphor converters. The sandwich structure with two filters and a phosphor converter enhanced the recycling of blue light and reduced its reflection loss, thus improving the PE to $180 \%$. Oh et al. [22] also proposed a similar sandwich structure with a filter, a phosphor converter and a polarizer. In this way, the luminous flux of the phosphor converters with this structure was increased to $139 \%$, and the correlated color temperature (CCT) consistency was better.

Although previous research indicated that structures or films on converters function well for improving PE, the scattering ability of micro-nanophosphor particles was also investigated. Fujita et al. [23] investigated the influence of the size of phosphor particles on luminous efficacy in Ce: $\mathrm{Y}_{3} \mathrm{Al}_{5} \mathrm{O}_{12}-\mathrm{Ce}^{3+}$ (YAG) phosphor-in-ceramics phosphor. They found that the luminous efficacy increased with particle size for the lowering scatter coefficient. According to Mie scatter theory, the cross section of microparticles in a unit volume decides the scattering coefficient [23-25]. In another aspects, Yang et al. [26] used a high density phosphor to increase center-light intensity. The scatter coefficient of phosphor with higher density was stronger compared with that of a lower density; thus, the input laser was trapped in the center of the phosphor. Zhou et al. [27] investigated the effects of particle size and density on optical performance of phosphor converters, and their results showed that a high concentration and low particle size contributed to large scatter coefficient. The work above reveals that particle size and density have a significant effect on phosphor converters and that the scatter characteristics are also a key parameter for the optical performance of phosphor converters.

The configurations for the high PE and luminous flux mentioned above also engender heat accumulation because the more luminous flux that is generated the more heat is transformed due to nonideal quantum efficiency. Therefore, laser lighting still faces the problem of heat dissipation under high-power density. For the laser light source, a variety of heat dissipation methods have been developed, including microchannels, heat pipes, forced air cooling, and double heat sinks [28-32]. Though, at present, there are reliable means to dissipate the heat of a laser light, phosphor converters are still facing the challenge of heat dissipation. Trivellin et al. [33] studied the failure of laser-lighting base materials and found that when the power of the irradiated point exceeded the power intensity limit (550 mW/0.1145 $\left.\mathrm{mm}^{2}\right)$, the material carbonized. Ding et al. [34] found that, though an $\mathrm{Al}$ substrate was used for heat dissipation, the phosphor converters with high concentration and large thickness still carbonized on the upper surface. To reduce heat accumulation, Narendran et al. [35] proposed dispersing the phosphors in the heat conduction matrix to form a regional phosphor structure, but its ultimate thermal conductivity was limited to that of the polymer materials. Ma et al. [36] proposed reducing concentration and thickness to improve temperature stability based on the study of temperature distribution in the polymer-based phosphor converters, but this led to a decrease in optical efficiency. Materials with high thermal conductivity and stability, such as glass and ceramics, contributed to improving thermal performance $[37,38]$. However, high fabrication costs and brittleness limit their application. Nevertheless, it is an alternative way to use a silicone converter with good heat dissipatio4n and optical performance for laser lighting device.

In addition, to the best of our knowledge, once the converter is manufactured, its lighting effects, such as CCT, luminous flux, cannot be changed. Therefore, based on the above-mentioned research, mainly in improving thermal stability and luminous flux, a new type of lighting effects-adjustable and hybrid lighting system with enhanced luminous flux and heat dissipation using a micro tunable angle filter is proposed. In this work, the filter separates blue and yellow light to reduce yellow reabsorption loss and reuse residual blue light. Experimental results showed that the lighting effects of the micro-angle tunable tilted filters (ATFPCs) are adjustable using the filter tilt angles, and the luminous flux and the heat dissipation are enhanced. 


\section{Materials and Methods}

\subsection{Characteristics of the Optical Filters}

The optical filters were manufactured by Shenzhen Giai Photonics Co., Ltd. (Shenzhen, Guangdong province, China) The filter is reflective, which allows long-wavelength light to pass through and reflects short-wavelength light, as shown in Figure 1a. For a filter, its cut-off frequency is bule-shifted when incident angle increases. The cut-off frequency of $545 \mathrm{THz}$ (wavelength: $550 \mathrm{~nm}$ ) guarantees that light with a lower wavelength will always be reflected when the incident angle increases, so a $550 \mathrm{~nm}$ filter was adopted. The characteristics of its transmission and reflection curves are shown in Figure 1b. Filter reflectance of light with a wavelength of less than $550 \mathrm{~nm}$ is greater than $92 \%$, and the transmittance of light with a wavelength of more than $550 \mathrm{~nm}$ is greater than $92 \%$.

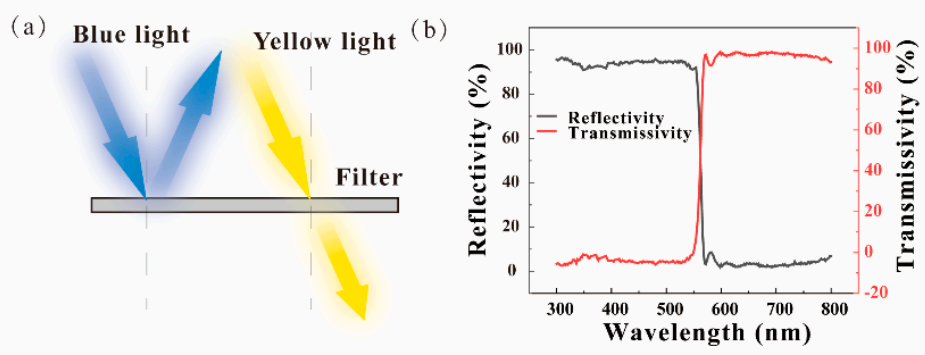

Figure 1. (a) Schematic diagram of the filter; (b) index of reflectance and transmission versus wavelength.

\subsection{Fabrication of $Y A G$ Yellow Phosphor Converters}

The $\mathrm{Y}_{3} \mathrm{Al}_{5} \mathrm{O}_{12}-\mathrm{Ce}^{3+}$ (YAG) yellow phosphor was manufactured by Shenzhen Zhangwanglong Technology Co. Ltd. (Shenzhen, Guangdong province, China). Polydimethylsiloxane (PDMS, manufactured by Shanghai Aladdin Bio-Chem Technology Co., Ltd (Shanghai, China) was used to encapsulate the phosphor particles. First, PDMS A and crosslink agent $B$ were mixed in a mass ration of 10:1. Second, the phosphor was added to prepare a mixture of $10 \%$ phosphor. Third, the mixture was stirred in a vacuum defoamer for about $5 \mathrm{~min}$. Fourth, the mixture of phosphor and PDMS was injected into premade modules with a thickness between 0.25 and $2 \mathrm{~mm}$, with an interval of $0.25 \mathrm{~mm}$. Finally, the samples were kept in an oven at $90{ }^{\circ} \mathrm{C}$ for $30 \mathrm{~min}$ for the matrix to solidify completely. The mass friction of the second step was adjusted from 10 to $30 \%$ with an interval of $2.5 \%$. The four steps above were repeated to prepare phosphor converters with different concentration of YAG: $\mathrm{Ce}^{3+}$.

\subsection{Experiment Setups}

As shown in Figure 2c, the ATFPC set up consisted of four parts-a PDMS-based phosphor converter, square filter, phosphor converter fixture with a hole and filter fixture with an angle scale that rotated around its own central axis as shown in Figures 2a and 3. The rotation angle of the filter fixture could be precisely controlled. The phosphor converter was tied to, and fixed on, the converter fixture and covered the hole. The two fit closely. The phosphor light passed through the hole, and the blue light and part of phosphor were reflected back to the phosphor layer; thus, a hybrid-type lighting system was formed by the transmission characteristics. The filter was fixed on the angle-tunable optical fixture, the central axis of which coincided with the rotation axis of the latter; thus, the filter was angle tunable when the fixture rotated. In each experiment, the height of the fixture axis and distance between the hole and converters remained unchanged. As a reference, a phosphor conversion system was also set up with a phosphor converter and two fixtures with no filter as shown in Figure 2b,d. All the elements of the ATFPCs were fixed on a small optical platform as shown in Figure 3. 
(a)

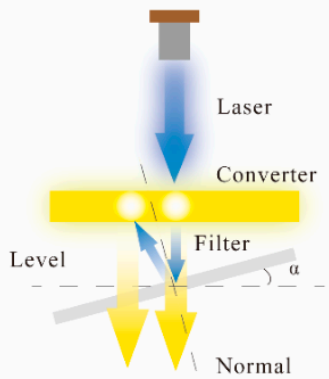

(c)

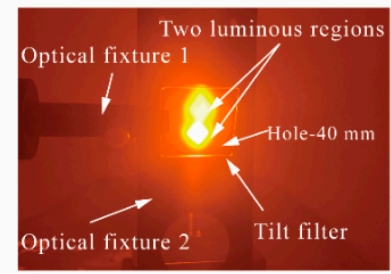

(b)

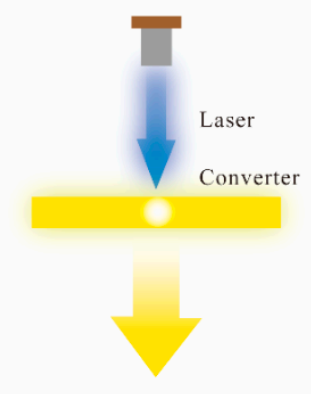

(d)

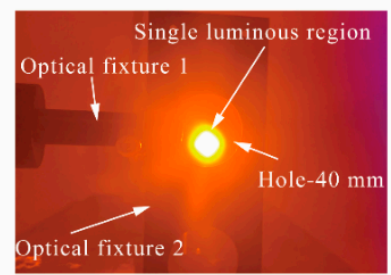

Figure 2. Diagrams of (a) micro-angle tunable tilted filters (ATFPCs) and (b)conventional phosphor converters (CPCs); actual lighting effect images of (c) CPCs and (d) ATFPCs under a brown filter to protect the camera.

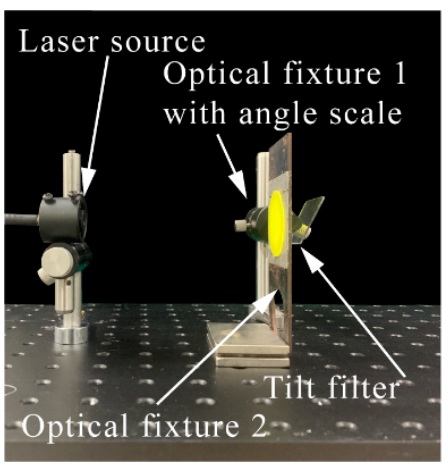

Figure 3. Configurations of the phosphor converters and actual lighting effects of the CPCs and ATFPCs.

The spectra of different laser-driven phosphor converters were experimentally investigated in a $900 \mathrm{~mm}$ diameter integrating sphere. All the phosphor systems were placed in it apart from the laser source which input the laser into the integrating sphere through an optical fiber. An optical detector was linked to a spectrometer from Ocean Insight (Dunedin, FL, USA). The thermal performance test of the ATFPC and CPC samples were carried out at room temperature by an infrared (IR) camera from FLIR Systems Inc., (Boston, MA, USA). The IR camera focused on the front surface to measure the temperature of the phosphor converters. In addition, the images were obtained by Zeiss's MERLIN scanning electron microscope (SEM) (Jena, Thuringia, Germany). The scattering characteristics of the phosphor particles was obtained through a spatial photometer, which had a measurement range from -90 to $90^{\circ}$.

\section{Results and Discussion}

\subsection{Scatter Characteristics of Phosphor Converters}

The SEM images of the phosphor particles were first explored to determine their sizes and morphology. Figure $4 \mathrm{a}$ is the images of phosphor particles at different magnifications. It shows irregular morphology with a mean particle size of $15 \mu \mathrm{m}$. Correspondingly, we obtained the scattering characteristics of these particles with different concentration and thickness using the spectrometer. In Figure $4 \mathrm{~b}$, the normalized intensity of scattered light 
by the phosphor converters tended to be smooth with increasing concentration from 17.5 to $30 \%$. A large light intensity still remains in the center positions. Since the scattering characteristics of particles partly depend on the concentration of the particles [23-27], greater concentration means a more uniform luminous intensity distribution. The variation of scattering characteristics with concentration is helpful for analyzing the gain effect of the micro-angle tunable tilted filter.

(a)

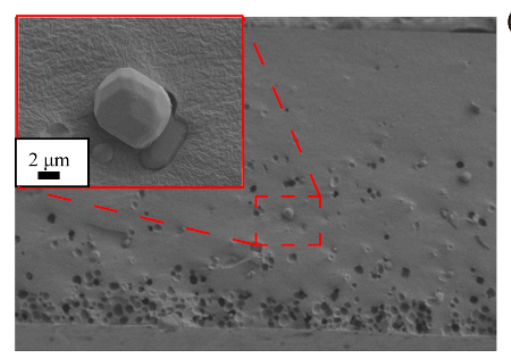

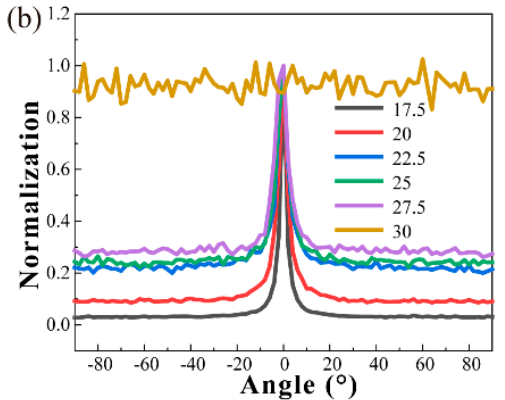

Figure 4. (a) The scanning electron microscope (SEM) images of microphosphor particles; (b) the scatter characteristics of phosphor converters with different concentrations.

\subsection{Effects of Tilt Angles on Optical Performance}

Considering the influence of angles on the luminous flux and the CCT of the ATFPCs, the concentration was fixed at $10 \%$ and the thickness at $0.5 \mathrm{~mm}$ to ensure a single variable. The power density of the laser was $10 \mathrm{~W} / \mathrm{cm}^{2}$, below the power density limit in [33]. Figure 5a shows the spectra of the ATFPCs with different tilt angles and CPCs. In the yellow light band (480-780 nm), the intensity of the ATFPCs rises in the range of $0-20^{\circ}$ and then falls, which is always higher than that of the CPCs. In the blue light band (380-480 nm), the intensity of the CPCs was the highest. Then the spectra and sub-spectra were integrated to obtain the total luminous flux and that of the residual blue and yellow light as shown in Figure 5 b,c. In Figure 5 b, the total luminous flux of the ATFPCs is superior to that of the CPCs. The relative increase in luminous flux of ATFPCs over CPCs kept rising in the range of $0-20^{\circ}$ and reached maximum value $11.5 \%$ at $20^{\circ}$. From 20 to $30^{\circ}$, although the luminous flux of ATFPCs decreased gradually, it still showed advantages over the CPCs. Figure $5 \mathrm{c}$ shows that the flux of residual ATFPC blue light decreased to $36.3 \%$ and the flux of yellow light increased to $207.4 \%$ compared with the CPCs. It is worth noting that the CCT of the phosphor converters increased from 6200 to $7100 \mathrm{~K}$ and it reached $6500 \mathrm{~K}$ at $20^{\circ}$, which was close to the standard white light source, as shown in Figure $5 \mathrm{~d}$. The CCT and luminous flux changed with the filter tilt angle, indicating that the lighting effects of AFTPCs were tunable. Furthermore, the luminous flux of the ATFPCs with tilt $20^{\circ}$ angle was the highest. The CCTs were also the closest to that of standard white source. The corresponding International Commission on illumination (CIE) chromaticity coordinates is presented as Table 1 and Figure 6a in detail. The title "WTO" in Table 1 means a CPC without a tilt filter.

Table 1. CIE coordinates verse tilt angle.

\begin{tabular}{ccccccccc}
\hline $\begin{array}{c}\text { CIE } \\
\text { Coordinates }\end{array}$ & $\mathbf{0}^{\circ}$ & $\mathbf{5}^{\circ}$ & $\mathbf{1 0}^{\circ}$ & $\mathbf{1 5}^{\circ}$ & $\mathbf{2 0}^{\circ}$ & $\mathbf{2 5}^{\circ}$ & $\mathbf{3 0}^{\circ}$ & WTO \\
\hline $\mathrm{x}$ & 0.3176 & 0.3154 & 0.3154 & 0.3138 & 0.3114 & 0.3083 & 0.3024 & 0.2419 \\
$\mathrm{y}$ & 0.3465 & 0.3428 & 0.3424 & 0.3413 & 0.3374 & 0.3334 & 0.3242 & 0.1973 \\
$\mathrm{z}$ & 0.3359 & 0.3418 & 0.3422 & 0.3449 & 0.3512 & 0.3582 & 0.3734 & 0.5609 \\
\hline
\end{tabular}


(a)

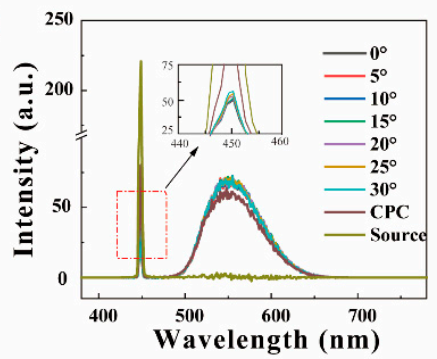

(c)

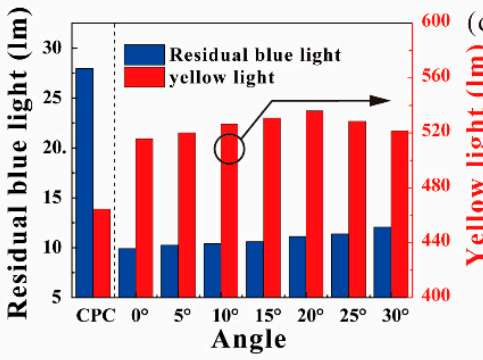

(b)
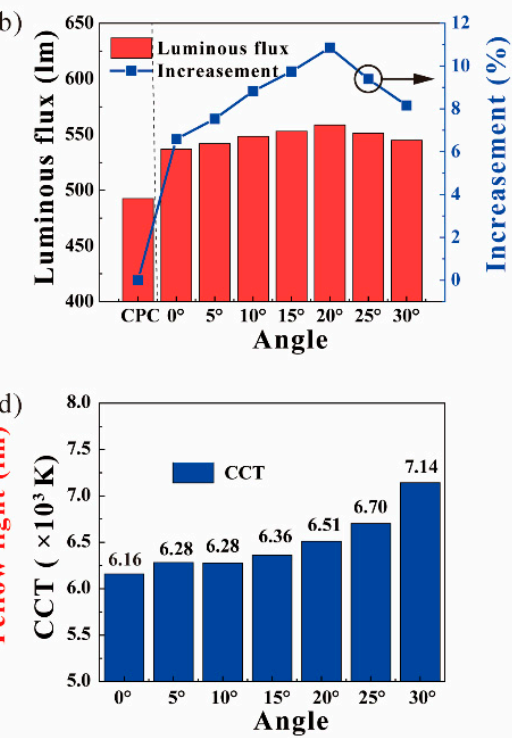

Figure 5. Effects of the title angle of the filter, spectra of (a) the CPCs, the ATFPCs; (b) luminous flux and increase in the ATFPCs over the CPCs; (c) residual bule rays and yellow light; (d) the correlated color temperature (CCT) changed with the title angle $\alpha$.

(a)

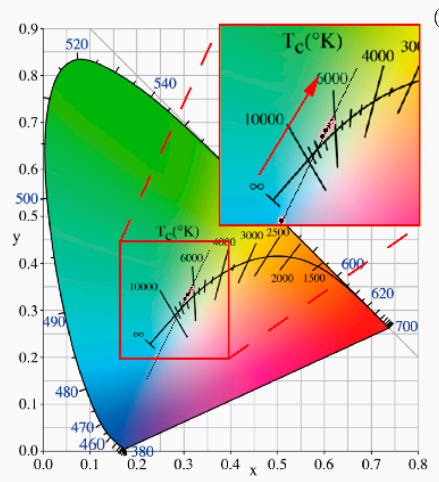

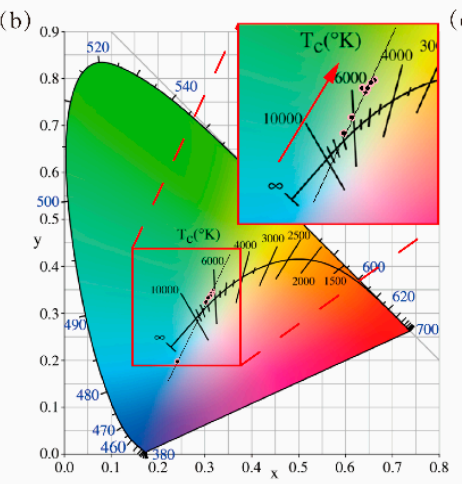

Figure 6. International Commission on illumination (CIE) chromaticity diagrams of (a) ATFPCs with different angles; (b) ATFPCs with different concentration; (c) CPCs with different concentration.

The increase in luminous flux resulted from the reuse of blue light excitation and is determined by the tilt angle $\alpha$. When the concentration of the phosphor converters is only $10 \%$, the phosphor particles in the directly illuminated area of high energy density laser are saturated, as shown in Figure 2d. Even if the remaining excited light returned along the original path to the emitting area, the enhancement of the light flux was limited. When the angle increased, a new excitation region was separated from the original one, which excited more phosphors in the unsaturated or unexcited state as shown in Figure 2c. Therefore, with the increasing angle, the effects were gradually enhanced. However, the distance between the two emitting regions positively correlated with the tangent value of the filter inclination. When the angle increased to a certain value, the residual blue light was too dispersed to shine completely on the phosphor converters, leading to increased residual blue light intensity. Then the luminous flux decreased and the CCT kept rising. It showed that the lighting effects of the ATFPCs could be adjusted by adjusting the inclination of the filter.

\subsection{Effects of Concentration on Optical Performance}

It is already known that the lighting effects are tunable using the tilt angle and that the effects are the best at $20^{\circ}$ (Figure 5). To further the optical and thermal performance 
of the ATFPCs optimize, the tilt angle $20^{\circ}$ was chosen in this section to continue the optimization. The laser power density was set to $10 \mathrm{~W} / \mathrm{cm}^{2}$. The thickness of converters was $0.5 \mathrm{~mm}$. Figure 7 discusses the effects of the phosphor converter concentration on the enhancement of the luminous flux and the CCT. The advantages of the ATFPCs over the CPCs in lighting effects were also analyzed. As shown in Figure 7a,b, the spectra of the ATFPCs and the CPCs indicated that the intensity in blue light band decreases with increasing concentration. But in yellow light band, the trend was just the opposite. By integrating the spectra, the luminous flux of the CPCs and the ATFPCs was obtained in Figure 7c. The results showed that the total flux of the CPCs increased with increasing concentration, and ATFPCs were similar. Moreover, the luminous flux of ATFPCs with $10 \%$ phosphor concentration was $98.2 \%$ of the CPC flux with $20 \%$ concentration. Therefore, with the ATFPC configuration, only half of the phosphor dose achieved the same luminous flux, which saves precious rare earth phosphors. But when the concentration rose to $30 \%$, the luminous flux of the ATFPCs was lower than that of the CPCs with the same concentration. Therefore, the dominant range of ATFPC concentration was 10-27.5\%. The increase in ATFPCs over CPCs also decreased with increasing concentration-from 16.4\% with $10 \%$ concentration to $-5 \%$ with $30 \%$ concentration as shown in Figure 7 c. Figure 7 indicates that, for the CPCs, if it was required to reach standard white light of about $6500 \mathrm{~K}$, the phosphor concentration needed to be increased to $25 \%$. In contrast, the ATPFCs achieved that with only $10 \%$ phosphor concentration. This proved that, compared to CPCs, ATPFC required a lower phosphor concentration, which benefited from the improved filter light conversion. The corresponding CIE chromaticity coordinates are presented as Tables 2 and 3, and Figure 6b,c. They show the linear relationship between the CIE chromaticity coordinates of ATFPCs and CPCs with different concentrations.

(a)

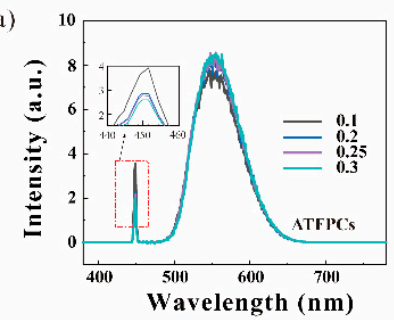

(c)

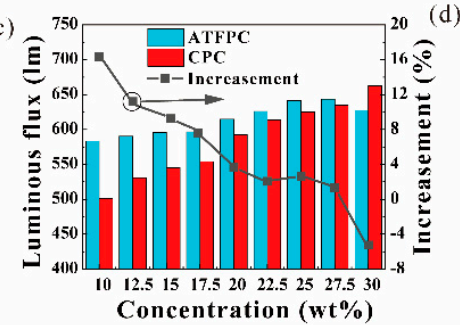

(b)
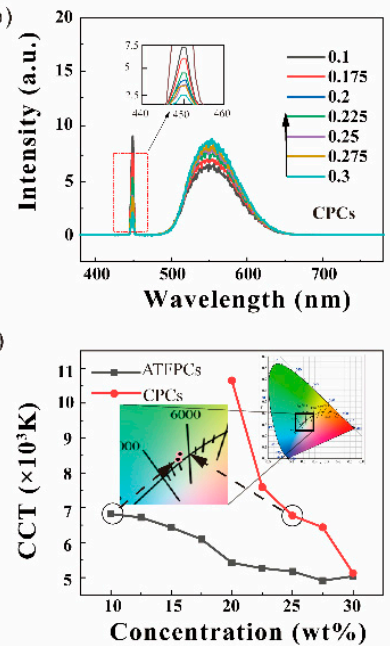

Figure 7. Effects of the concentration of phosphor particles, spectra of (a) CPCs and (b) ATFPCs; (c) luminous flux and relative increase in ATFPCs; (d) the CCT of ATFPCs and CPCs.

Table 2. CIE coordinates verse phosphor concentration of ATFPCs.

\begin{tabular}{cccccccc}
\hline $\begin{array}{c}\text { CIE } \\
\text { Coordinates }\end{array}$ & $\mathbf{1 0} \mathbf{w t} \%$ & $\mathbf{1 7 . 5} \mathbf{w t} \%$ & $\mathbf{2 0} \mathbf{w t} \%$ & $\mathbf{2 2 . 5} \mathbf{w t} \%$ & $\mathbf{2 5} \mathbf{w t} \%$ & $\mathbf{2 7 . 5} \mathbf{w t} \%$ & $\mathbf{3 0} \mathbf{w t} \%$ \\
\hline $\mathrm{x}$ & 0.3071 & 0.3185 & 0.3401 & 0.335 & 0.3433 & 0.3533 & 0.3486 \\
$\mathrm{y}$ & 0.3279 & 0.3522 & 0.3907 & 0.3821 & 0.3961 & 0.4083 & 0.4044 \\
$\mathrm{z}$ & 0.365 & 0.3294 & 0.2692 & 0.2829 & 0.2606 & 0.2383 & 0.2471 \\
\hline
\end{tabular}


Table 3. CIE coordinates verse phosphor concentration of CPCs.

\begin{tabular}{cccccccc}
\hline $\begin{array}{c}\text { CIE } \\
\text { Coordinates }\end{array}$ & $\mathbf{1 0} \mathbf{w t} \%$ & $\mathbf{1 7 . 5} \mathbf{w t} \%$ & $\mathbf{2 0} \mathbf{w t} \%$ & $\mathbf{2 2 . 5} \mathbf{w t} \%$ & $\mathbf{2 5} \mathbf{w t} \%$ & $\mathbf{2 7 . 5} \mathbf{w t} \%$ & $\mathbf{3 0} \mathbf{w t} \%$ \\
\hline $\mathrm{x}$ & 0.2381 & 0.2537 & 0.2979 & 0.2806 & 0.3074 & 0.3456 & 0.3123 \\
$\mathrm{y}$ & 0.1891 & 0.2213 & 0.3145 & 0.2787 & 0.3315 & 0.4075 & 0.3415 \\
$\mathrm{z}$ & 0.5728 & 0.525 & 0.3876 & 0.4408 & 0.3611 & 0.2469 & 0.3462 \\
\hline
\end{tabular}

When the phosphor concentration rose, the phosphor particles had a strong scattering ability to the laser, as shown in Figure $4 \mathrm{~b}$. After the first time the blue laser penetrated the phosphor converters, the residual laser had been fully scattered and the central light intensity was quite low. Therefore, the filter in the ATFPCs had a problem reguiding all the residual blue light back to the converters. In addition, the filter absorbed the blue and yellow light to a certain extent. Finally, the gain brought by the reuse of blue light by the filter was smaller than the loss caused by its absorption. Thus, ATFPCs performed than CPCs in luminous flux with high concentration. Instead ATFCs had more significant advantages in the low-concentration fluorescence range.

\subsection{Thermal Performance of ATFPCs}

It is already known from the above analysis that ATFPCs with $10 \%$ concentration performed better both in luminous flux and the CCT compared with the CPCs whether with the same concentration $(10 \%)$ or double $(20 \%)$. The heat dissipation of the ATFPCs needed to be verified to explore further the application potential of the ATFPCs. The concentration of the ATFPCs was 10\%, that of the CPCs 10\% (denoted as CPC \#1) and $20 \%$ (denoted as CPC \#2). In this experiment, the laser power density was $10 \mathrm{~W} / \mathrm{cm}^{2}$, and the thickness of the ATFPCs and CPCs was $0.5 \mathrm{~mm}$. Figure 8 shows the difference in temperature distribution of the ATFPCs over CPC \#1 versus tilt angle $\alpha$. Here, heat dissipation is characterized by maximum temperature (MT). As shown in Figure 8, the maximum surface ATFPC temperature decreased by $11.7 \%$ from 77.7 to $69.5^{\circ} \mathrm{C}$ with an increasing tilt angle. Compared with CPC \#1, the increase in the maximum temperature of the ATFPCs was up to $15.9 \%$ at $0^{\circ}$, and as low as $1.6 \%$ at $30^{\circ}$. The temperature increase in the ATFPCs was caused by the secondary use of the laser. When the inclination angle was $20^{\circ}$, the MT of the ATFPC was $7.2 \%$ higher than that of CPC \#1. Meanwhile, the PE of the ATFPCs improved 11.5\% compared to that of the CPCs as shown in Figure 8f, which verified the above hypothesis.

Furthermore, the temperature distribution of the ATFPC with a $20^{\circ}$ tilted angle was compared with the CPC under the same luminous flux. Therefore, CPC \#2 with the luminous flux as large as ATFPC's was also analyzed in Figure 9. The MT of the ATFPC and the $\mathrm{CPC}$ was 67 and $93^{\circ} \mathrm{C}$, respectively. Although the PE of the two was similar, the ATFPC temperature fell by $21.2{ }^{\circ} \mathrm{C}$, which is $22.8 \%$ lower. This showed that our proposed strategy had excellent heat dissipation performance under the same luminous flux.

The high concentration and large thickness of the phosphor converters absorbed and transformed the blue light in the direct irradiation area and its adjacent area during the laser first incidence, but it was accompanied with a lot of heat generation. At the same time, the thermal conductivity of the polymer-based phosphor converters was poor, resulting in heat accumulation in a small area, and finally a carbonized surface. Low-concentration and small-thickness phosphor converters can reduce the local MT by reducing the conversion and absorption of the incident blue light, but it also means low luminous flux and high CCT. This thorny contradiction can be effectively resolved through the proposed ATFPC strategy. When such converters are equipped with a micro-angle tunable tilted filter, the filter reflects the residual blue light passing through the ATFPCs in another direction, so that the high-intensity blue light energy can be dispersed, thereby improving the heat dissipation of the ATFPCs. 

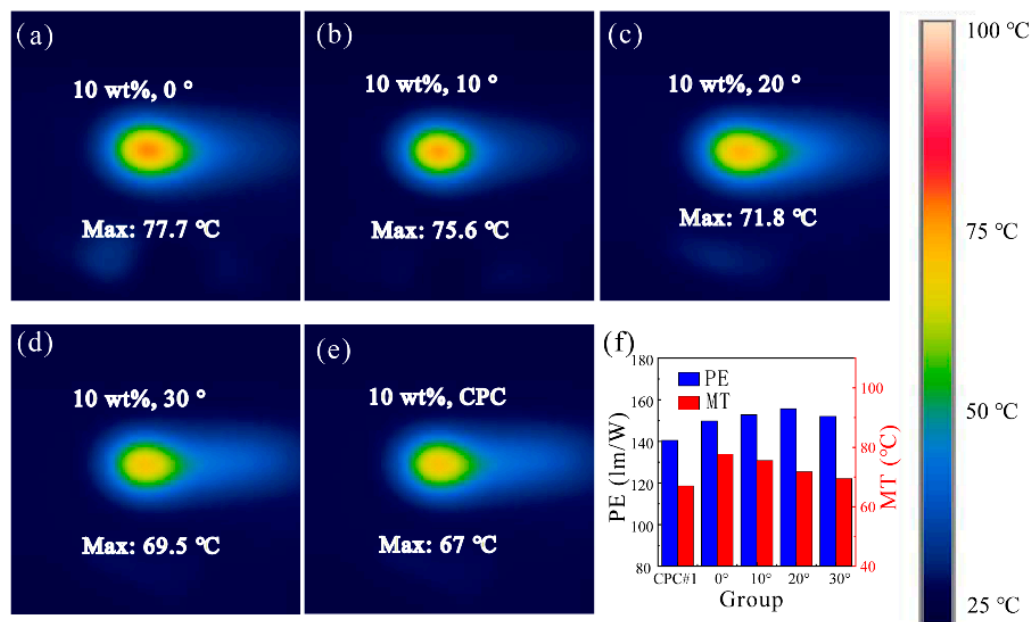

$50{ }^{\circ} \mathrm{C}$

Figure 8. Temperature distribution of ATFPCs with (a) $10 \%$ concentration, $0^{\circ}$; (b) $10 \%$ concentration, $10^{\circ}$; (c) $10 \%$ concentration, $20^{\circ}$; (d) $10 \%$ concentration, $30^{\circ}$ and the CPCs with (e) $10 \%$ concentration; and photoluminescence efficiency (PE) of (f) the ATFPCs as well as the CPCs, with the same thickness of $0.5 \mathrm{~mm}$.
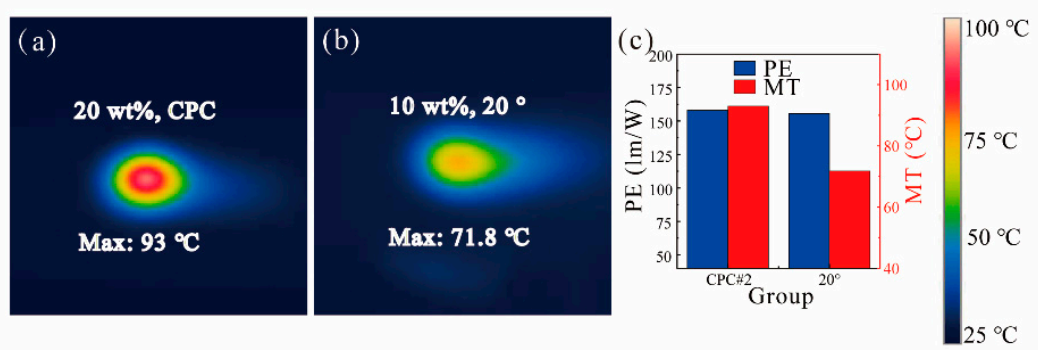

Figure 9. Temperature distribution of (a) the ATFPC; (b) the CPC; (c) PE of (a) the ATFPC; (b) the CPC.

\subsection{Intensity Regulation of Uneven Lumination}

To further determine and optimize the intensity distribution of ATFPCs, a simulation model of ATFPCs at $20^{\circ}$ was set up and run by TracePro as shown in Figure 10d. The ATFPC was placed at 40 on the $z$ axis. A parabolic reflector with a 40 focal length was placed at the coordinate origin. An object plane was placed at 200 on the $z$ axis. Figure 10 indicates the real and simulated light intensity distribution of ATFPCs at a $20^{\circ}$ tilt angle. In Figure $9 \mathrm{a}$, there are two peaks of intensity distribution curves of ATFPCs at -50 and $20^{\circ}$, in the $0-180^{\circ}$ semi-planar. The peak at $50^{\circ}$ is the nonideal peak of the leaked bule light and the green dot curve is ideal with no leaked blue light. The saddle shaped light distribution is undesirable for lighting. Without the reflector, the simulation results of the module are shown in Figure 10b. It is consistent with the related true color images with $505.18 \mathrm{~lm}$ flux in Figure 10e. It shows that there are two peaks of the curve in the $0-180^{\circ}$ semi-planar. The results were similar to those in Figure 10b, meaning that the simulation is reasonable. To couple the two peaks, the parabolic reflector was introduced. The curves in Figure 10c tended to be smoother and more suitable for lighting, especially for headlights. Its true color images with $508.35 \mathrm{~lm}$ flux are shown in Figure 10f. 


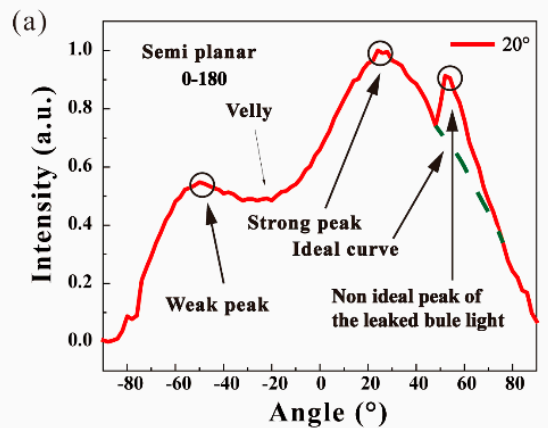

(c)

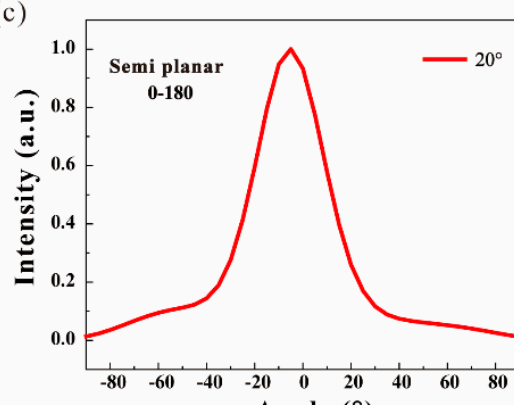

Angle ( $\left.{ }^{\circ}\right)$

(e)

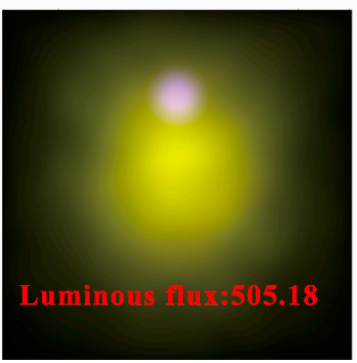

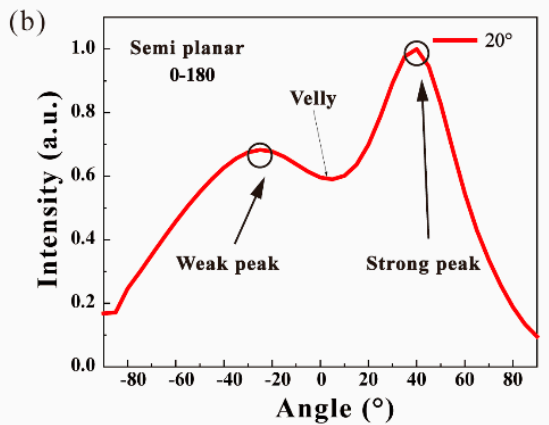

(d)

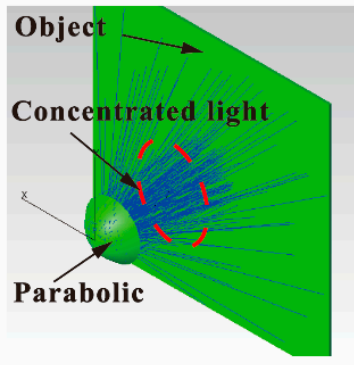

(f)

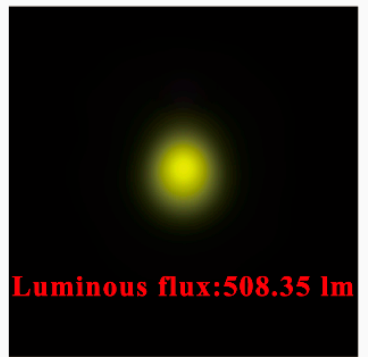

Figure 10. Light intensity distribution of (a) experiments, simulations (b) before and (c) after optimization; (d) simulation models of modulated ATFPCs, true color images of ATFPCS (e) without and (f) with a reflector.

The intensity distribution of ATFPCs was uneven due to the reguiding of the microangle tunable tilted filter. In the normal of the filter, the intensity was stronger than in the direction of incidence. Therefore, there were two peaks with different intensities in the two directions. When the parabolic reflector was introduced, the weak and strong peaks were gathered to the center and coupled to each other. Hence, the valley in two peaks disappeared and a new peak occurred at $10^{\circ}$, indicating that the reshaping of intensity distribution curves is easily done without extra luminous flux loss.

\section{Conclusions}

This work proposed a new type of adjustable laser lighting system with enhanced luminous flux and heat dissipation using a tunable angle to recycle laser. Optical and thermal performances and light distribution were experimentally or simultaneity investigated, respectively. Taking advantage of the tilted angle, the reflective filter reguided residual blue light to another region on the same phosphor converter after the first incidence and partial absorption; thus, two luminous regions were formed and the energy of the laser was reused. The results showed that the lighting effects of the ATFPCs, such as luminous flux and CCT, can be adjusted by tuning the tilt angle of the filter. The optimization of the spatial distribution of intensity was discussed as well. At $20^{\circ}$, the ATFPCs we proposed had $11.5 \%$ more luminous flux and increased photoluminescence efficiency compared with CPCs with the same concentration. Its CCT was closer to that of a standard white light source as well. Under the same luminous flux, though, the CCT of the two types of 
converters were all about $6500 \mathrm{~K}$, and the concentration of the ATFPCs is just half of that of the CPCs. The maximum temperature of the ATFPCs was also $22.8 \%$ lower than for the CPCs. Based on the above conclusions, ATFPCs provide a new heat-dissipation solution for laser lighting systems, paving the way for their wide application.

Author Contributions: Conceptualization, X.D.; methodology, C.Y.; investigation, C.Y.; resource, L.X.; data curation, J.L.; writing-original draft preparation, R.Q.; writing-review \& editing, X.D.; visualization, R.Q.; supervision, B.Y.; project administration, B.Y.; funding acquisition, Z.L. All authors have read and agreed to the published version of the manuscript.

Funding: This work was performed by National and Local Joint Engineering Research Center of Semiconductor Display and Optical Communication Devices, South China University of Technology and supported by the National Natural Science Foundation of China (No. 51805173, 52075186), the Natural Science Foundation of Guangdong Province (2019A1515011741), the Science and Technology Program of Guangzhou (201904010252), and The Project of the National and Local Joint Engineering Research Center of Semiconductor Display and Optical Communication (Zhongshan Branch) (190919172214566).

Conflicts of Interest: The authors declare no conflict of interest.

\section{References}

1. Chang, Y.P.; Chang, J.K.; Chen, H.A.; Chang, S.H.; Liu, C.N.; Han, P.; Cheng, W.H. An advanced laser headlight module employing highly reliable glass phosphor. Opt. Express 2019, 27, 1808-1815. [CrossRef] [PubMed]

2. Barnum, P.C.; Narasimhan, S.G.; Kanade, T. A multi-layered display with water drops. ACM Trans. Graph. 2010, 29, 1-7. [CrossRef]

3. Chang, Y.P.; Liu, C.N.; Pei, Z.; Lee, S.M.; Lai, Y.K.; Han, P.; Shih, H.K.; Cheng, W.H. New scheme of LiDAR-Embedded smart laser headlight for autonomous vehicles. Opt. Express 2019, 27, A1481-A1489. [CrossRef]

4. Barnum, P.; Narasimhan, S.; Kanade, T. A projector-camera system for creating a display with water drops. In Proceedings of the 2009 IEEE Computer Society Conference on Computer Vision and Pattern Recognition Workshops (IEEE2009), Miami, FL, USA, 20-25 June 2009; pp. 17-23.

5. Wolf, A.; Kloppenburg, G.; Danov, R.; Lachmayer, R.J.D.P. DMD based automotive lighting unit. In DGaO Proceedings 2016; Deutschen Gesellschaft für angewandte Optik: Erlangen, Germany, 2016.

6. Lee, B.L.; Douglass, M.R.; Lachmayer, R.; Kloppenburg, G.; Knöchelmann, M. Headlamp innovations: Optical concepts for fully adaptive light distributions. In Emerging Digital Micromirror Device Based Systems and Applications X; International Society for Optics and Photonics: Bellingham, WA, USA, 2018; Volume 10546, p. 105460K.

7. Denault, K.A.; Cantore, M.; Nakamura, S.; DenBaars, S.P.; Seshadri, R. Efficient and stable laser-driven white lighting. AIP Adv. 2013, 3, 072107. [CrossRef]

8. Yuan, Y.; Wang, D.; Zhou, B.; Feng, S.; Sun, M.; Zhang, S.; Gao, W.; Bi, Y.; Qin, H. High luminous fluorescence generation using Ce: YAG transparent ceramic excited by blue laser diode. Opt. Mater. Express 2018, 8, 2760-2767. [CrossRef]

9. Gu, C.; Wang, X.-J.; Xia, C.; Li, S.; Liu, P.; Li, D.; Li, H.; Zhou, G.; Zhang, J.; Xie, R.-J. A new CaF $2-$ YAG: Ce composite phosphor ceramic for high-power and high-color-rendering WLEDs. J. Mater. Chem. C 2019, 7, 8569-8574. [CrossRef]

10. Hu, S.; Liu, Y.; Zhang, Y.; Xue, Z.; Wang, Z.; Zhou, G.; Lu, C.; Li, H.; Wang, S. 3D printed ceramic phosphor and the photoluminescence property under blue laser excitation. J. Eur. Ceram. Soc. 2019, 39, 2731-2738. [CrossRef]

11. Liu, Z.; Li, S.; Huang, Y.; Wang, L.; Zhang, H.; Jiang, R.; Huang, F.; Yao, X.; Liu, X.; Huang, Z. The effect of the porosity on the Al2O3-YAG: Ce phosphor ceramic: Microstructure; luminescent efficiency; and luminous stability in laser-driven lighting. $J$. Alloys Compd. 2019, 785, 125-130. [CrossRef]

12. Wang, H.; Mou, Y.; Peng, Y.; Zhang, Y.; Wang, A.; Xu, L.; Long, H.; Chen, M.; Dai, J.; Chen, C. Fabrication of phosphor glass film on aluminum plate by using lead-free tellurite glass for laser-driven white lighting. J. Alloys Compd. 2020, 814, 152331. [CrossRef]

13. Xu, J.; Yang, Y.; Wang, J.; Du, B.; Santamaría, A.A.; Hu, B.; Liu, B.; Ji, H.; Dam-Hansen, C.; Jensen, O.B. Industry-friendly synthesis and high saturation threshold of a LuAG: Ce/glass composite film realizing high- brightness laser lighting. J. Eur. Ceram. Soc. 2020, 40, 6031-6036. [CrossRef]

14. Li, S.; Tang, D.; Tian, Z.; Liu, X.; Takeda, T.; Hirosaki, N.; Xu, F.; Huang, Z.; Xie, R.-J. New insights into the microstructure of translucent $\mathrm{CaAlSiN}_{3}: \mathrm{Eu}^{2+}$ phosphor ceramics for solid-state laser lighting. J. Mater. Chem. C 2017, 5, 1042-1051. [CrossRef]

15. Wang, H.; Dai, J.; Sun, H.; Mou, Y.; Cai, Y.; Liang, R.; Xu, L.; Gao, Y.; Peng, Y.; Li, J.; et al. Phosphor Glass- Coated Sapphire with Moth-Eye Microstructures for Ultraviolet-Excited White Light-Emitting Diodes. IEEE Trans. Electron Devices 2019, 66, 3007-3011. [CrossRef]

16. Li, Z.-T.; Wang, Q.-H.; Tang, Y.; Li, C.; Ding, X.-R.; He, Z.-H. Light Extraction Improvement for LED COB Devices by Introducing a Patterned Lead frame Substrate Configuration. IEEE Trans. Electron Devices 2013, 60, 1397-1403. [CrossRef]

17. Ding, X.; Tang, Y.; Li, Z.; Li, J.; Xie, Y.; Lin, L. Multichip LED Modules with V-Groove Surfaces for Light Extraction Efficiency Enhancements Considering Roughness Scattering. IEEE Trans. Electron Devices 2017, 64, 182-188. [CrossRef] 
18. Pan, J.-W.; Wang, C.-S.J.O.e. Light extraction efficiency of GaN-based LED with pyramid texture by using ray path analysis. Opt. Express 2012, 20, 630-640. [CrossRef]

19. Fujii, T.; Gao, Y.; Sharma, R.; Hu, E.L.; DenBaars, S.P.; Nakamura, S. Increase in the extraction efficiency of GaN-based lightemitting diodes via surface roughening. Appl. Phys. Lett. 2004, 84, 855-857. [CrossRef]

20. Sun, B.; Zhao, L.; Wei, T.; Yi, X.; Liu, Z.; Wang, G.; Li, J.; Yi, F. Light extraction enhancement of bulk GaN light-emitting diode with hemisphere-cones-hybrid surface. Opt. Express 2012, 20, 18537-18544. [CrossRef]

21. Tang, Y.; Liang, Y.; Ding, X.; Wu, Q.; Yu, B. A laser-driven phosphor converted system with enhanced optical efficiency by using light-recycling dichroic filters. J. Lumin. 2020, 223, 117180. [CrossRef]

22. Oh, J.H.; Yang, S.J.; Do, Y.R. Polarized white light from LEDs using remote-phosphor layer sandwiched between reflective polarizer and light-recycling dichroic filter. Opt. Express 2013, 21, A765-A773. [CrossRef] [PubMed]

23. Fujita, S.; Umayahara, Y.; Tanabe, S.J. Influence of light scattering on luminous efficacy in Ce: YAG glass-ceramic phosphor. J. Ceram. Soc. Jpn. 2010, 118, 128-131. [CrossRef]

24. Kim, S.; Kim, H. Optical properties of phosphor-in-glass through modification of pore properties for LED packaging. Opt. Mater. 2018, 75, 814-820. [CrossRef]

25. Li, J.-S.; Chen, J.-X.; Lin, L.-W.; Li, Z.-T.; Tang, Y.; Yu, B.-H.; Ding, X.-R. A Detailed Study on Phosphor- Converted Light-Emitting Diodes with Multi-Phosphor Configuration Using the Finite-Difference Time-Domain and Ray-Tracing Methods. IEEE J. Quantum Electron. 2015, 51, 1-10. [CrossRef]

26. Yang, Y.; Long, T.; Zhuang, S.; Wang, L.; Luo, Y. High-Particle-Density YAG: Ce Phosphor Coating for High Power Laser Lighting. J. Electron. Packag. 2020, 142, 031101. [CrossRef]

27. Zhuo, N.; Zhang, N.; Jiang, T.; Chen, P.; Wang, H. Effect of particle sizes and mass ratios of a phosphor on light color performance of a green phosphor thin film and a laminated white light-emitting diode. RSC Adv. 2019, 9, 27424-27431. [CrossRef]

28. Murshed, S.M.S.; de Castro, C.A.N. A critical review of traditional and emerging techniques and fluids for electronics cooling. Renew. Sustain. Energy Rev. 2017, 78, 821-833. [CrossRef]

29. Beni, S.B.; Bahrami, A.; Salimpour, M.R. Design of novel geometries for microchannel heat sinks used for cooling diode lasers. Int. J. Heat Mass Transf. 2017, 112, 689-698. [CrossRef]

30. Kozłowska, A.; Łapka, P.; Seredyński, M.; Teodorczyk, M.; Dabrowska-Tumańska, E. Experimental study and numerical modeling of micro-channel cooler with micro-pipes for high-power diode laser arrays. Appl. Therm. Eng. 2015, 91, 279-287. [CrossRef]

31. Nozaki, S.; Yoshida, S.; Yamanaka, K.; Imafuji, O.; Takigawa, S.; Katayama, T.; Tanaka, T. High-power and high-temperature operation of an InGaN laser over $3 \mathrm{~W}$ at $85^{\circ} \mathrm{C}$ using a novel double-heat-flow packaging technology. Jpn. J. Appl. Phys. 2016, 55, 04EH05. [CrossRef]

32. Zhu, H.; Hao, M.; Zhang, J.; Ji, W.; Lin, X.; Zhang, J.; Ning, Y. Development and thermal management of $10 \mathrm{~kW}$ CW; direct diode laser source. Opt. Laser Technol. 2016, 76, 101-105. [CrossRef]

33. Trivellin, N.; Yushchenko, M.; Buffolo, M.; de Santi, C.; Meneghini, M.; Meneghesso, G.; Zanoni, E. Laser-Based Lighting: Experimental Analysis and Perspectives. Materials 2017, 10, 1166. [CrossRef]

34. Ding, X.; Li, M.; Li, Z.; Tang, Y.; Xie, Y.; Tang, X.; Fu, T. Thermal and optical investigations of a laser-driven phosphor converter coated on a heat pipe. Appl. Therm. Eng. 2019, 148, 1099-1106. [CrossRef]

35. Perera, I.U.; Narendran, N. Analysis of a remote phosphor layer heat sink to reduce phosphor operating temperature. Int. J. Heat Mass Transf. 2018, 117, 211-222. [CrossRef]

36. Ma, Y.; Lan, W.; Xie, B.; Hu, R.; Luo, X. An optical-thermal model for laser-excited remote phosphor with thermal quenching. Int. J. Heat Mass Transf. 2018, 116, 694-702. [CrossRef]

37. Peng, Y.; Sun, Q.; Liu, J.; Mou, Y.; Wang, X.; Chen, M.; Luo, X. Reflective Phosphor-in-Glass Color Converter for Laser-Driven White Lighting. IEEE Photonics Technol. Lett. 2020, 32, 983-986. [CrossRef]

38. Zheng, P.; Li, S.; Wang, L.; Zhou, T.L.; You, S.; Takeda, T.; Hirosaki, N.; Xie, R.J. Unique Color Converter Architecture Enabling Phosphor-in-Glass (PiG) Films Suitable for High-Power and High-Luminance Laser- Driven White Lighting. ACS Appl. Mater. Interfaces 2018, 10, 14930-14940. [CrossRef] 